\title{
Determination of Iridoid Glucosides for Quality Assessment of Herba Oldenlandiae by High-Performance Liquid Chromatography
}

\author{
Zhi-Tao Liang, Zhi-Hong Jiang, Kelvin Sze-Yin Leung, and Zhong-Zhen ZhaO* \\ School of Chinese Medicine, Hong Kong Baptist University; Kowloon Tong, Hong Kong, P. R. China. \\ Received March 7, 2006; accepted May 8, 2006
}

\begin{abstract}
Herba Oldenlandiae, the dried herb of Oldenlandia diffusa (WILLD.) RoxB. (Family Rubiaceae), is officially listed in the Chinese Pharmacopoeia. In the herbal market, two substitutes originated from $O$. corymbosa (L.) LAM and $O$. tenelliflora $B_{L}$. are commonly used. In light of this, the target in setting up a method for quality assessment of Herba Oldenlandiae is urgently needed. In this article, a simple and reliable high-performance liquid chromatographic (HPLC) method was developed for quantifying asperuloside (1), $E$-6- $O$-p-coumaroyl scandoside methyl ester (2) and $E$-6-O-p-coumaroyl scandoside methyl ester-10-methyl ether (3) in Herba Oldenlandiae derived from $O$. diffusa. Among them, compound 3 is a new compound isolated from $O$. diffusa. All these unique compounds were used as markers for the first time in the quality assessment of Herba Oldenlandiae. The results showed that the contents of compounds $1-3$ were significantly varied among different samples whilst those of compounds 2 and 3 were found to be lower in contents in the two substitutes of $O$. diffusa. The analytical method is suitable for quality control of Herba Oldenlandiae and useful in differentiation from its confusable species. The method has been fully validated with satisfactory linearity, accuracy, precision and stability.
\end{abstract}

Key words Oldenlandia; iridoid glucoside; Herba Oldenlandiae; quality control; HPLC

Herba Oldenlandiae, a well known folk medicine in China, has been used for the treatment of tonsillitis, sore throat, appendicitis and urethral infection since Qing Dynasty. At present, it is generally used in clinic to treat hepatitis and malignant tumors of the liver, as well as an ingredient in herbal tea in China. ${ }^{1)}$ Herba Oldenlandiae is also an important ingredient in a variety of Chinese herbal preparations, such as HuaHong tablet, Yi-Gan-Ning granule and Bai-Hua-She-SheCao Injection, etc. According to the Chinese Pharmacopoeia, Herba Oldenlandiae refers to the dried herb of Oldenlandia diffusa (WILlD.) RoxB. (Family Rubiaceae). ${ }^{2)}$ However, a systematic market investigation on Herba Oldenlandiae retrieved that in the wholesale and food markets of Hong Kong, Macau and Guangdong Province, O. corymbosa (L.) LAM has been substitutely used and also referred to as Herba Oldenlandiae. ${ }^{3)}$ Worst more, another species of $O$. tenelliflora $\mathrm{BL}$. has also been found to be used as Herba Oldenlandiae in Yunnan and Guangdong Provinces. These confusions in the market have led to a growing concern about the genuineness and the quality assessment needed for Herba Oldenlandiae.

Previous phytochemical studies of $O$. diffusa have demonstrated that Herba Oldenlandiae contains a majority of iridoid glucosides, triterpenoids, flavonoids and polysaccharides. ${ }^{4-10)}$ Among the iridoid glucosides, asperuloside (1) and $E$-6- $O-p$-coumaroyl scandoside methyl ester (2) have been isolated from $O$. diffusa. ${ }^{4,5)}$ In this paper, a new compound named $E$-6- $O$ - $p$-coumaroyl scandoside methyl ester10 -methyl ether (3) was isolated from this herb in our laboratory. In the literature, compound $\mathbf{1}$ was reported to be present in $O$. corymbosa but not $O$. tenelliflora, while compounds 2 and 3 were not found in either herb. ${ }^{1)}$ Although the activity of the three compounds has not been evaluated in current study, previous pharmacological studies showed that iridoid glucosides possessed the effects of anti-inflammatory, antioxidant, anti-angiogenic as well as properties of inhibiting allelochemicals and DNA polymerase, nerve growth factor-potentiating, anti-implantation, etc. ${ }^{11-18)}$
Despite the popularity of usage, relevant quality control method for Herba Oldenlandiae is very limited. In the literatures, quality assessment of Herba Oldenlandiae always relied on ursolic acid and oleanolic acid as the marker compounds. ${ }^{19-21)}$ Besides, the content of polysaccharide, total flavone or $p$-coumaric acid has also been used for quality assessment index. ${ }^{22,23)}$ However, ursolic acid and oleanolic acid were commonly found in other plants and not specified enough for the identification of $O$. diffusa. Moreover, the contents of the two compounds varied slightly between $O$. diffusa and $O$. corymbosa. ${ }^{19)}$ Therefore, these two chemical compounds are not ideal candidates for the quality assessment of Herba Oldenlandiae. Iridoid glucosides, the main components of $O$. diffusa, should be considered as chemical markers for the quality assessment purpose. However, related studies have not been conducted until now. The present work focused on developing a rapid, facile and reliable quantitative method for determination of the three iridoid glucosides, asperuloside (1), E-6-O-p-coumaroyl scandoside methyl ester (2) and E-6-O-p-coumaroyl scandoside methyl ester-10methyl ether (3). The relative contents will be used to evaluate the quality of Herba Oldenlandiae and differentiate among substitutes (Fig. 1).

\section{Experimental}

Plant Materials The whole dried herb, used for extraction and isolation the compounds $\mathbf{1}-\mathbf{3}$, and was purchased in Guangdong province, China, in 2005. Twenty-three samples of $O$. diffusa (WILLD.) RoxB., sixteen samples of $O$. corymbosa (L.) LAM and four samples of $O$. tenelliflora BL. were purchased from herbal markets or collected from the cultivation area in China (Tables 1,2). Sample batch numbers 19 to 22 of $O$. diffusa and 6 to 9 of $O$. corymbosa were respective samples collected from the same herbal market over six consecutive months. Batches 1 to 6 of $O$. corymbosa were collected from the same herbal market over five consecutive months. All the herbs were authenticated by the authors and the corresponding voucher specimen were deposited in the Bank of China (HK) Chinese Medicines Centre of Hong Kong Baptist University.

Instrumentation Melting points were determined using a Barnstead Electrothermal IA9100 instrument. IR spectra were recorded in $\mathrm{KBr}$ using a Perkin Elmer TG/DTA spectrometer. NMR spectra were recorded with a 


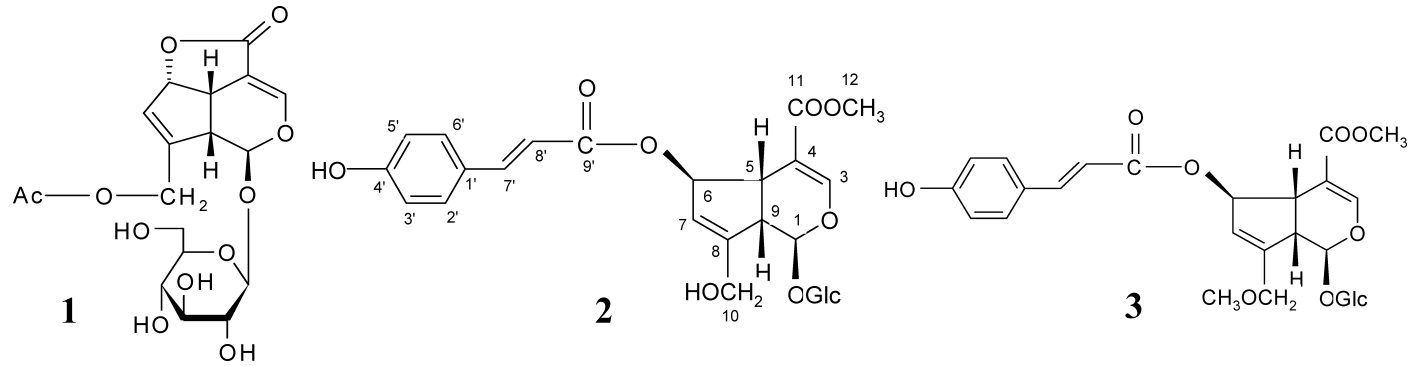

Fig. 1. Chemical Structures of Compounds $\mathbf{1}-\mathbf{3}$

Table 1. Contents (mg/g) of Iridoid Glucosides in the Samples of Oldenlandia diffusa (WILLD.) RoxB.

\begin{tabular}{|c|c|c|c|c|c|}
\hline Sample No. & Source & Collecting time & Compound $\mathbf{1}^{a)}$ & Compound 2 & Compound $\mathbf{3}$ \\
\hline 1 & Yibin, Sichuan; field & 2004.08 .01 & $7.885 \pm 0.286$ & $5.135 \pm 0.307$ & ${ }^{b)}$ \\
\hline 2 & Chongqing, Sichuan; market & 2004.07 .25 & - & $1.183 \pm 0.025$ & - \\
\hline 3 & Yixing, Jiangsu; cultivated & 2004.08 .16 & $1.466 \pm 0.015$ & $1.104 \pm 0.018$ & $1.135 \pm 0.002$ \\
\hline 4 & Nanjing, Jiangsu; market & 2004.08 .15 & $0.569 \pm 0.018$ & $2.703 \pm 0.088$ & $0.549 \pm 0.033$ \\
\hline 5 & Hefei, Anhui; market & 2004.08 .17 & $1.142 \pm 0.027$ & $3.700 \pm 0.006$ & $0.209 \pm 0.002$ \\
\hline 6 & Fuan, Fujian; field & 2005.08 .21 & $1.874 \pm 0.056$ & $5.525 \pm 0.003$ & trace \\
\hline 7 & Putian, Fujian; field & 2005.08 .21 & $1.033 \pm 0.037$ & $7.159 \pm 0.023$ & trace \\
\hline 8 & Fuzhou, Fujian; field & 2005.08 .21 & $9.869 \pm 0.070$ & $6.117 \pm 0.053$ & - \\
\hline 9 & Kunming, Yunnan; market & 2004.08 .24 & - & $1.970 \pm 0.029$ & - \\
\hline 10 & Nanning, Guangxi; field & 2005.07 .04 & $7.185 \pm 0.019$ & $6.875 \pm 0.010$ & $1.077 \pm 0.001$ \\
\hline 11 & Nanning, Guangxi; market & 2005.07 .04 & $0.412 \pm 0.032$ & $3.506 \pm 0.005$ & trace \\
\hline 12 & Ruyuan, Guangdong; field & 2004.07.09 & $5.217 \pm 0.028$ & $6.311 \pm 0.063$ & trace \\
\hline 13 & Gaoyao, Guangdong; field & 2004.09 .02 & $1.188 \pm 0.092$ & $3.527 \pm 0.032$ & $0.267 \pm 0.003$ \\
\hline 14 & Guangzhou, Guangdong; market & 2005.03 .24 & $1.812 \pm 0.071$ & $5.109 \pm 0.213$ & $1.227 \pm 0.061$ \\
\hline 15 & Luoding, Guangdong; field & 2005.08 .19 & $1.646 \pm 0.049$ & $3.400 \pm 0.185$ & $0.328 \pm 0.025$ \\
\hline 16 & Luoding, Guangdong; market & 2005.08 .24 & $0.199 \pm 0.001$ & $3.347 \pm 0.078$ & $0.078 \pm 0.005$ \\
\hline 17 & Beijing; market & 1999.07.08 & $0.589 \pm 0.018$ & $4.275 \pm 0.091$ & $0.303 \pm 0.017$ \\
\hline 18 & Shenzhen, Guangdong; cultivated & 2005.10 .30 & $5.051 \pm 0.083$ & $2.849 \pm 0.080$ & $1.313 \pm 0.057$ \\
\hline 19 & Luoding, Guangdong; market (batch: LD0517) & 2005.05 .17 & $0.370 \pm 0.004$ & $3.940 \pm 0.047$ & $0.930 \pm 0.006$ \\
\hline 20 & Luoding, Guangdong; market (batch: LD0601) & 2005.06 .01 & $0.581 \pm 0.004$ & $4.978 \pm 0.039$ & $0.889 \pm 0.011$ \\
\hline 21 & Luoding, Guangdong; market (batch: LD0616) & 2005.06 .16 & $1.622 \pm 0.005$ & $6.071 \pm 0.015$ & $1.795 \pm 0.006$ \\
\hline 22 & Luoding, Guangdong; market (batch: LD0901) & 2005.09 .01 & $1.338 \pm 0.011$ & $2.826 \pm 0.005$ & $0.970 \pm 0.012$ \\
\hline 23 & Lushan, Jiangxi; field & 2005.10 .18 & $0.948 \pm 0.064$ & $4.214 \pm 0.034$ & - \\
\hline Mean \pm S.D. & & & $2.476 \pm 2.804$ & $4.166 \pm 1.703$ & $0.791 \pm 0.509$ \\
\hline
\end{tabular}

a) $\mathrm{mg} / \mathrm{g}$, mean \pm S.D. $(n=2) ; b)(-)$ below detection limit.

Table 2. Contents (mg/g) of Iridoid Glucosides in the Samples of Oldenlandia corymbosa (L.) Lam and O. tenelliflora BL.

\begin{tabular}{|c|c|c|c|c|c|c|}
\hline Sample No. & Latin name & Source & Collecting time & Compound $\mathbf{1}^{a}$ & Compound 2 & Compound $\mathbf{3}$ \\
\hline 1 & O. corymbosa & Hong Kong; market (batch: LF06) & 2005.06.19 & - & - & ${ }^{b)}$ \\
\hline 2 & O. corymbosa & Hong Kong; market (batch: LF07) & 2005.07.19 & $0.770 \pm 0.011$ & - & - \\
\hline 3 & O. corymbosa & Hong Kong; market (batch: LF08) & 2005.08 .19 & $0.482 \pm 0.001$ & - & - \\
\hline 4 & O. corymbosa & Hong Kong; market (batch: LF09) & 2005.09 .19 & $1.521 \pm 0.099$ & - & - \\
\hline 5 & O. corymbosa & Hong Kong; market (batch: LF10) & 2005.10 .19 & $3.363 \pm 0.052$ & $0.183 \pm 0.003$ & - \\
\hline 6 & O. corymbosa & Luoding, Guangdong; market (batch: LD0701) & 2005.07 .01 & $0.751 \pm 0.006$ & - & - \\
\hline 7 & O. corymbosa & Luoding, Guangdong; market (batch: LD0715) & 2005.07.15 & $0.770 \pm 0.011$ & - & - \\
\hline 8 & O. corymbosa & Luoding, Guangdong; market (batch: LD0731) & 2005.07 .31 & $0.657 \pm 0.005$ & - & - \\
\hline 9 & O. corymbosa & Luoding, Guangdong; market (batch: LD0815) & 2005.08 .15 & - & - & - \\
\hline 10 & O. corymbosa & Shenzhen, Guangdong; cutivated & 2005.10 .30 & $2.946 \pm 0.098$ & - & - \\
\hline 11 & O. corymbosa & Wutongzai, Hong Kong; field & 2004.08.09 & $0.248 \pm 0.215$ & $0.135 \pm 0.003$ & trace \\
\hline 12 & O. corymbosa & Hong Kong; market & 2004.10 .23 & $5.063 \pm 0.009$ & - & - \\
\hline 13 & O. corymbosa & Gaoyao, Guangdong; market & 2004.09 .02 & trace & $0.367 \pm 0.005$ & trace \\
\hline 14 & O. corymbosa & Guangzhou, Guangdong; market & 2004.08 .26 & trace & - & - \\
\hline 15 & O. corymbosa & Nanning, Guangxi; field (from Qingxiu Mountain) & 2005.07 .04 & $2.156 \pm 0.007$ & $0.402 \pm 0.015$ & - \\
\hline 16 & O. corymbosa & $\begin{array}{l}\text { Nanning, Guangxi; field (from Guangxi Botanical } \\
\text { Garden of Medicinal Plants) }\end{array}$ & 2005.07 .04 & $1.625 \pm 0.074$ & - & - \\
\hline Mean \pm S.D. & & & & $1.690 \pm 0.272$ & $1.452 \pm 0.132$ & \\
\hline 17 & O. tenelliflora & Haixiawan, Hong Kong; field & 2004.10 .09 & $1.33 \pm 0.036$ & - & - \\
\hline 18 & O. tenelliflora & Yibin, Sichuan; field & 2004.08 .03 & $0.300 \pm 0.001$ & - & - \\
\hline 19 & O. tenelliflora & Kunming, Yunnan; market & 2004.08 .24 & - & - & - \\
\hline 20 & O. tenelliflora & Wongyuan, Guangdong; field & 2005.11 .10 & $1.170 \pm 0.042$ & - & - \\
\hline Mean \pm S.D. & & & & $0.933 \pm 0.554$ & & \\
\hline
\end{tabular}

a) $\mathrm{mg} / \mathrm{g}$, mean \pm S.D. $(n=2) ; b)(-)$ below detection limit. 
JEOL JNM-LA400 (400 MHz for ${ }^{1} \mathrm{H}$; $100 \mathrm{MHz}$ for $\left.{ }^{13} \mathrm{C}\right)$ spectrometer using TMS as internal standard. High-resolution mass spectrum was measured on a Bruker Daltonics Autoflex MALDI-TOF mass spectrometer. A CREST 1875HTAG ultrasonic processor (CREST, U.S.A.) was used for sample extraction.

An Agilent 1100 series HPLC system equipped with a G1311A Quaternary Pumps, a G1315A Dio-Array Detector, a G1322A Degasser and a G1313A autosampler was used. An Applied Biosystems/PE-SCIEX API 365 LC-MS system with electrospray ionization source (Applied Biosystems, U.S.A.) was used for mass spectrometric measurements. A Zorbax Eclipse XDB-C18 column $(250 \mathrm{~mm} \times 4.6 \mathrm{~mm}, 5 \mu \mathrm{m}$, Agilent Technologies, U.S.A.) coupled with a $\mathrm{C} 18$ guard column $(7.5 \mathrm{~mm} \times 4.6 \mathrm{~mm}, 5 \mu \mathrm{m}$, Alltech Associates, U.S.A.) was used at room temperature. The mobile phase was a mixture of water (A) and acetonitrile (B) at a flow-rate of $1.0 \mathrm{ml} / \mathrm{min}$. The gradient elution of mobile phase was $12 \% \mathrm{~B}$ in $0-5 \mathrm{~min}, 12-20 \% \mathrm{~B}$ in $5-12 \mathrm{~min}, 20 \% \mathrm{~B}$ in $12-25 \mathrm{~min}$ and $20-35 \% \mathrm{~B}$ in $25-35 \mathrm{~min}$. The detection wavelength was set at $238 \mathrm{~nm}$ and $312 \mathrm{~nm}$ with a reference wavelength of $550 \mathrm{~nm}$. UV spectra of compounds $\mathbf{1}-\mathbf{3}$ were acquired from 200 to $400 \mathrm{~nm}$.

Chemicals and Reagents General grade petroleum ether 60-80 (International Laboratory, U.S.A.), analytical grade methanol, chloroform, ethyl acetate and $n$-butanol (Labscan, Bangkok, Thailand) were used for extraction and isolation of compounds $\mathbf{1}-\mathbf{3}$. Column chromatography was performed over Silica gel 60 (70-230 mesh, Merck) or reversed-phase octadecyl silica (ODS) (Fuji Sylysia Chemical Ltd., Japan).

HPLC grade acetonitrile (Labscan, Bangkok, Thailand) and deionized water obtained from a Milli-Q water system (Millipore, Bedford, MA, U.S.A.) were used for preparation of mobile phase. Analytical grade methanol (Labscan, Bangkok, Thailand) was used for preparation of standard and sample extraction. The reference compounds of asperuloside (1), $E$-6-O-p-coumaroyl scandoside methyl ester (2) and E-6-O-p-coumaroyl scandoside methyl ester-10-methyl ether (3) were isolated and purified from the dried herb of Oldenlandia diffusa (WILLD) RoxB. in our laboratory. Their structures were elucidated on the basis of HR-MS, ${ }^{1} \mathrm{H}$ - and ${ }^{13} \mathrm{C}-\mathrm{NMR}$ spectral data. The purity of the three components determined by HPLC-UV was shown to be greater than $97 \%$.

Extraction and Isolation of the Compounds $1-\mathbf{3}$ The dried whole herbs $(5.6 \mathrm{~kg})$ of $O$. diffusa were extracted with 281 methanol for $1 \mathrm{~h}$ in an ultrasonic processor for twice. The extracts were combined and concentrated under reduced pressure. The residue $(419.3 \mathrm{~g})$ was dissolved in water and successively partitioned with petroleum ether layer $(54.8 \mathrm{~g})$, chloroform layer $(39.4 \mathrm{~g})$, ethyl acetate layer $(14.9 \mathrm{~g})$ and $n$-butanol layer $(61.6 \mathrm{~g})$. The chloroform extract was further fractionated by silica gel column chromatography using a mobile phase of petroleum ether/ethyl acetate of increasing polarity ( $100 \%$ petroleum ether $\rightarrow 100 \%$ ethyl acetate) to obtain 15 fractions. The fraction $13(5.2 \mathrm{~g})$ was eluted with petroleum ether and ethyl acetate $(90 \%$ petroleum ether $\rightarrow 50 \%$ petroleum ether) over silica gel column. Subfractions 5, 7 and 9 derived from fraction 13 were further purified on an ODS column eluted with 70,60 and $60 \%$ methanol to give compound 3 (10 mg), 2 (15 mg) and 1 (8 mg), respectively.

The ethyl acetate extract was fractionated by column chromatography over silica gel using a $\mathrm{CHCl}_{3}$ and $\mathrm{MeOH}$ mixture of increasing polarity to give 12 fractions (from 2 to $50 \% \mathrm{MeOH}$ ). Fractions 7, 9 and 11 were further purified on an ODS column eluted with 70,60 and 60\% methanol to give compound $\mathbf{3}$ (385.2 $\mathrm{mg}), 2$ (1849 $\mathrm{mg}$ ) and $\mathbf{1}$ (240.5 mg), respectively.

The $n$-butanol extract ( $28 \mathrm{~g}$ ) was chromatographed over silica gel using a $\mathrm{CHCl}_{3}$ and $\mathrm{MeOH}$ mixture of increasing polarity to give 15 combined fractions $\left(100 \% \mathrm{CHCl}_{3}\right.$ to $\left.100 \% \mathrm{MeOH}\right)$. Compound 1 (1091 mg) was obtained from fraction 5 by crystallization.

Preparation of Standard Solution The three iridoid glucosides $(\mathbf{1}-\mathbf{3})$ were accurately weighed and then dissolved in methanol to produce stock standard solutions at a concentration of about $1000 \mu \mathrm{g} / \mathrm{ml}$ which were kept at $4{ }^{\circ} \mathrm{C}$. Calibration curves were established with nine concentrations within the range of $5.0-1000 \mu \mathrm{g} / \mathrm{ml}$ for each iridoid glucoside by appropriate dilution of the stock solution with methanol.

Preparation of Sample Solution Samples were ground into powder and passed through a 20 -mesh $(0.9 \mathrm{~mm})$ sieve. $0.5 \mathrm{~g}$ sample powder was accurately weighed and transferred into a $50 \mathrm{ml}$ centrifuge tube. Each sample was extracted twice with $25 \mathrm{ml}$ of $80 \%$ methanol by sonication for $60 \mathrm{~min}$ at room temperature. After centrifugation at $3000 \mathrm{rpm}$ for $5 \mathrm{~min}$, the supernatant was transferred into $50 \mathrm{ml}$ volumetric flask and was adjusted to the volume with $80 \%$ methanol. The solution was then filtered through a $0.45 \mu \mathrm{m}$ membrane. An aliquot of $20 \mu \mathrm{l}$ solution was subjected to HPLC analysis.

\section{Results and Discussion}

Elucidation of the Structures of Compounds 1-3 The compounds $\mathbf{1} \mathbf{- 2}$ were determined to be asperuloside (1) and $E$-6-O-p-coumaroyl scandoside methyl ester (2) by comparing their physical, ${ }^{1} \mathrm{H}$ - and ${ }^{13} \mathrm{C}-\mathrm{NMR}$ spectral data with previously reported data. ${ }^{4,5,7)}$

Compound 3 was obtained as slightly yellow powder; $\mathrm{mp}$ $59-60^{\circ} \mathrm{C} ; \lambda_{\max } 312(\varepsilon 3365)(\mathrm{MeOH})$. The molecular formula was determined to be $\mathrm{C}_{27} \mathrm{H}_{32} \mathrm{O}_{13}$ by HR-MS $(\mathrm{m} / \mathrm{z}$ 587.1764 of $[\mathrm{M}+\mathrm{Na}]^{+}$, calculated for $\mathrm{C}_{27} \mathrm{H}_{32} \mathrm{O}_{13} \mathrm{Na}$, theoretical value: 587.1740$)$. It showed absorption bands at 3399 $(\mathrm{OH}), 1708(\mathrm{C}=\mathrm{O}), 1632,1605(\mathrm{C}=\mathrm{C}), 1514$ and $835 \mathrm{~cm}^{-1}$ (benzenoid) in the IR spectrum. The ${ }^{1} \mathrm{H}-\mathrm{NMR}$ spectrum revealed two carbomethoxy singlets at $\delta 3.79\left(\mathrm{OCH}_{3}\right)$ and $\delta$ $3.56\left(\mathrm{OCH}_{3}\right)$, which were consistent with the ${ }^{13} \mathrm{C}-\mathrm{NMR}$ data. A proton signal of $\mathrm{C}-3$ of iridoid's main structure was evident from a singlet at $\delta 7.45(1 \mathrm{H})$. In addition, signals at $\delta 6.43$ and $\delta 7.59$ due to a simple $\mathrm{AB}$ system $(J=15.9 \mathrm{~Hz})$ characteristic of trans olefinic protons; signals at $\delta 7.65$ and $\delta 6.96$ were due to an $\mathrm{A}_{2} \mathrm{~B}_{2}$ system $(J=8.7 \mathrm{~Hz})$. These data indicate the presence of $p$-coumaroyl group in compound 3. Compare with those spectral data of the known compound 2 (Tables 3 , 4), compound 3 was determined to have an additional methoxy group signal. ${ }^{4,5,7)}$ Therefore, the structure of $\mathbf{3}$ is de-

Table 3. ${ }^{1} \mathrm{H}-\mathrm{NMR}$ Data of Compounds $\mathbf{2}$ and $\mathbf{3}$

\begin{tabular}{lll}
\hline \multicolumn{1}{c}{ No. } & \multicolumn{1}{c}{$\mathbf{3}\left(\delta^{a)}\right)$} & \multicolumn{1}{c}{$\mathbf{2}(\delta)$} \\
\hline $\mathrm{H}-1$ & $5.28, \mathrm{~d}, J=6.0 \mathrm{~Hz}^{a)}$ & $5.30, \mathrm{~d}, J=6.0 \mathrm{~Hz}$ \\
$\mathrm{H}-3$ & $7.45, \mathrm{~s}$ & $7.50, \mathrm{~s}$ \\
$\mathrm{H}-5$ & $3.16, \mathrm{~d}, J=9.0 \mathrm{~Hz}$ & $3.20, \mathrm{~d}, J=9.0 \mathrm{~Hz}$ \\
$\mathrm{H}-6$ & $5.56, \mathrm{~m}$ & $5.67, \mathrm{~m}$ \\
$\mathrm{H}-7$ & $5.73, \mathrm{~m}$ & $5.85, \mathrm{~m}$ \\
$\mathrm{H}-9$ & $2.50, \mathrm{~m}$ & $3.11, \mathrm{~m}$ \\
$\mathrm{H}-10$ & $4.20, \mathrm{~d}, J=15.9 \mathrm{~Hz}$ & $4.39, \mathrm{~d}, J=15.6 \mathrm{~Hz}$ \\
$\mathrm{H}-10$ & $4.04, \mathrm{~d}, J=15.9 \mathrm{~Hz}$ & $4.22, \mathrm{~d}, J=15.6 \mathrm{~Hz}$ \\
$\mathrm{H}-12$ & $3.56, \mathrm{~s}$ & $3.65, \mathrm{~s}$ \\
$\mathrm{H}-2^{\prime}, 6^{\prime}$ & $7.65, \mathrm{~d}, J=8.7 \mathrm{~Hz}$ & $7.46, \mathrm{~d}, J=8.4 \mathrm{~Hz}$ \\
$\mathrm{H}-3^{\prime}, 5^{\prime}$ & $6.96, \mathrm{~d}, J=8.7 \mathrm{~Hz}$ & $6.80, \mathrm{~d}, J=8.4 \mathrm{~Hz}$ \\
$\mathrm{H}-7^{\prime}$ & $7.59, \mathrm{~d}, J=15.9 \mathrm{~Hz}$ & $7.62, \mathrm{~d}, J=15.9 \mathrm{~Hz}$ \\
$\mathrm{H}-8^{\prime}$ & $6.43, \mathrm{~d}, J=15.9 \mathrm{~Hz}$ & $6.33, \mathrm{~d}, J=15.9 \mathrm{~Hz}$ \\
glc-1 & $4.51, \mathrm{~d}, J=7.8 \mathrm{~Hz}$ & $4.69, \mathrm{~d}, J=7.8 \mathrm{~Hz}$ \\
glc-2, $3,4,5$ & $2.97-3.24(4 \mathrm{H}, \mathrm{m})$ & $3.20-3.42(4 \mathrm{H}, \mathrm{m})$ \\
glc-6 & $3.68, \mathrm{~d}, J=11.1 \mathrm{~Hz}$ & $3.88, \mathrm{~d}, J=11.1 \mathrm{~Hz}$ \\
$-\mathrm{OCH}_{3}$ & $3.79, \mathrm{~s}$ & \\
& &
\end{tabular}

a) $\delta$ : chemical shift, Hz: coupling constant.

Table $4 .{ }^{13} \mathrm{C}-\mathrm{NMR}$ Data of Compounds $\mathbf{2}$ and $\mathbf{3}$

\begin{tabular}{|c|c|c|c|c|c|}
\hline No. & $3\left(\delta^{a}\right)$ & $2(\delta)$ & No. & $3(\delta)$ & $2(\delta)$ \\
\hline C-1 & 96.1 & 97.8 & $\mathrm{C}-3^{\prime}, 5^{\prime}$ & 116.1 & 116.7 \\
\hline C-3 & 153.0 & 153.9 & C-4' & 161.7 & 161.0 \\
\hline C-4 & 108.8 & 109.8 & $C-7^{\prime}$ & 144.9 & 146.4 \\
\hline C-5 & 40.7 & 42.3 & C-8' & 115.1 & 115.3 \\
\hline C-6 & 82.3 & 83.5 & C-9' & 166.2 & 168.6 \\
\hline C-7 & 125.4 & 127.1 & glc-1 & 99.2 & 100.2 \\
\hline C-8 & 150.5 & 150.1 & glc-2 & 73.9 & 74.7 \\
\hline C-9 & 46.3 & 46.9 & glc-3 & 77.9 & 78.3 \\
\hline C-10 & 59.7 & 60.9 & glc-4 & 70.7 & 71.4 \\
\hline C-11 & 167.2 & 168.9 & glc-5 & 77.2 & 77.8 \\
\hline C-12 & 51.9 & 52.0 & glc-6 & 61.8 & 62.6 \\
\hline C-1' & 127.3 & 127.1 & $\mathrm{OCH}_{3}$ & 56.1 & \\
\hline $\mathrm{C}-2^{\prime}, 6^{\prime}$ & 130.7 & 131.0 & & & \\
\hline
\end{tabular}

a) Chemical shift. 
termined to be $E-6-O-p$-coumaroyl scandoside methyl ester10 -methyl ether which was not reported previously.

Identification of Compounds $\mathbf{1}-\mathbf{3}$ in Chromatogram and Selection of Measurement Wavelength Apart from comparing with the corresponding retention time, compounds $\mathbf{1}-\mathbf{3}$ were identified by UV features and LC-ESI-MS analysis in order to provide further information on their identities. Protonated ion of asperuloside (1), E-6-O-p-coumaroyl scandoside methyl ester (2) and E-6-O-p-coumaroyl scandoside methyl ester-10-methyl ether (3) were obtained at 437.0, 573.2 and $587.0 \mathrm{~m} / \mathrm{z}$, respectively together with their corresponding UV spectra (Fig. 2). In general, the chemical markets identified in LC-ESI-MS and UV spectra for standard references and the corresponding samples were found to be consistent.

In the UV spectra, absorption maxima were observed for compound $\mathbf{1}$ at $238 \mathrm{~nm}$ whilst $312 \mathrm{~nm}$ for compounds $\mathbf{2}$ and 3. Therefore, these two wavelengths were selected for HPLC analysis of the three components.

Extraction Condition The choices of extraction solvents and time were optimized. Each of the herbal samples was extracted by ultrasonication for $60 \mathrm{~min}$ using a list of solvents in various concentrations: $95 \%$ ethanol, absolute methanol, 80,70 and $50 \%$ methanol. The results showed that $80 \%$ methanol produced the best yield for compounds $\mathbf{1}$ and $\mathbf{2}$ while a reasonable high yield for compounds 3 (Table 5). The extraction efficiency was further optimized in terms of extraction time at $30,90,120,150$ and 180 min with $80 \%$ methanol. The results indicated that a prolonged extraction time gave better extraction efficiency (Table 6). However, this also bears the risk of sample deterioration as a result of heat generated by sonication. These conditions were optimized with extraction for twice and comparable extraction efficiency. The extraction methods other than sonication were also investigated. In general, sonication was found to be better than extraction under reflux and soxhlet in terms of ex-
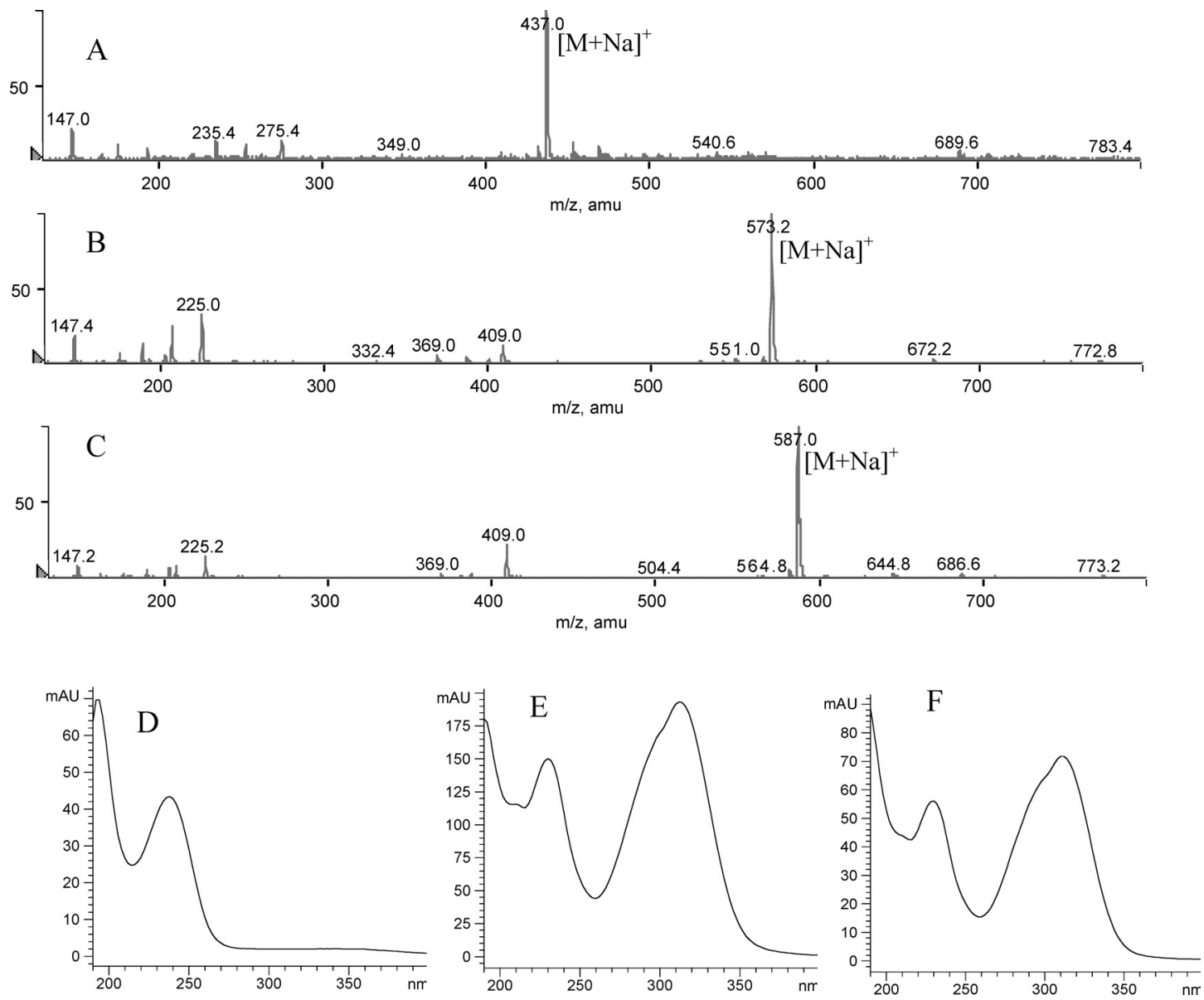

Fig. 2. On-Line Detected Mass Spectra and UV Spectra of Compounds $1(A, D), 2(B, E)$ and $3(C, F)$

Table 5. Selection of Extraction Solvent for Efficient Extraction of Compounds 1-3 in Herbal Samples

\begin{tabular}{cccccr}
\hline \hline \multirow{2}{*}{ Compound } & \multicolumn{3}{c}{ Extraction solvent } \\
\cline { 2 - 6 } & $95 \%$ ethanol $^{a)}$ & Methanol & $80 \%$ methanol & $70 \%$ methanol & $50 \%$ methanol \\
\hline $\mathbf{1}$ & $0.127 \pm 0.016$ & $0.425 \pm 0.028$ & $0.665 \pm 0.014$ & $0.460 \pm 0.007$ & $0.622 \pm 0.026$ \\
$\mathbf{3}$ & $1.198 \pm 0.066$ & $3.140 \pm 0.109$ & $3.187 \pm 0.042$ & $2.302 \pm 0.237$ & $2.977 \pm 0.086$ \\
& $0.095 \pm 3.25 \mathrm{E}-05$ & $0.235 \pm 0.010$ & $0.234 \pm 0.007$ & $0.167 \pm 0.021$ & $0.0206 \pm 0.002$ \\
\hline
\end{tabular}

a) $\mathrm{mg} / \mathrm{g}$, mean \pm S.D. $(n=2)$. 
Table 6. Influences of Extraction Time on Extraction Efficiency for Compounds 1-3

\begin{tabular}{|c|c|c|c|c|c|}
\hline \multirow{2}{*}{ Compound } & \multicolumn{5}{|c|}{ Extraction time (min) } \\
\hline & $30^{a)}$ & 90 & 120 & 150 & 180 \\
\hline 1 & $0.338 \pm 0.029$ & $0.588 \pm 0.010$ & $0.630 \pm 0.014$ & $0.639 \pm 0.010$ & $0.614 \pm 0.030$ \\
\hline 2 & $1.943 \pm 0.0002$ & $3.629 \pm 0.037$ & $3.738 \pm 0.148$ & $4.061 \pm 0.036$ & $4.095 \pm 0.067$ \\
\hline 3 & $0.140 \pm 0.002$ & $0.243 \pm 0.001$ & $0.254 \pm 0.007$ & $0.280 \pm 0.017$ & $0.283 \pm 0.004$ \\
\hline
\end{tabular}

a) $\mathrm{mg} / \mathrm{g}$, mean \pm S.D. $(n=2)$

Table 7. Extraction Methods for Compounds $\mathbf{1}-\mathbf{3}$

\begin{tabular}{cccc}
\hline \hline \multirow{2}{*}{ Compound } & \multicolumn{3}{c}{ Extraction method } \\
\cline { 2 - 4 } & Ultrasonication $^{a)}$ & Reflux & Soxhlet $^{b)}$ \\
\hline $\mathbf{1}$ & $0.589 \pm 0.018$ & $0.702 \pm 0.048$ & $0.575 \pm 0.017$ \\
$\mathbf{2}$ & $4.275 \pm 0.091$ & $3.931 \pm 0.159$ & $4.249 \pm 0.151$ \\
$\mathbf{3}$ & $0.303 \pm 0.017$ & $0.270 \pm 0.014$ & $0.302 \pm 0.015$ \\
\hline
\end{tabular}

a) $\mathrm{mg} / \mathrm{g}$, mean \pm S.D. $(n=2) ; b)$ the extraction solvent is methanol (considering the principle of Soxhlet) and the extraction time is $5 \mathrm{~h}$.
Table 8. Linear Regression and Correlation Coefficients for Compounds 1-3

\begin{tabular}{cccc}
\hline \hline Compound & $\begin{array}{c}\text { Linear range } \\
(\mu \mathrm{g} / \mathrm{ml})\end{array}$ & $\begin{array}{c}\text { Regression } \\
\left.\text { equation }^{a}\right)\end{array}$ & $\begin{array}{c}\text { Correlation } \\
\text { coefficient }\left(R^{2}\right)\end{array}$ \\
\hline $\mathbf{1}$ & $5.28-1056$ & $y=4.389 x+10.916$ & 0.9996 \\
$\mathbf{3}$ & $5.36-1066$ & $y=9.0258 x+10.897$ & 1.0000 \\
$\mathbf{3}$ & $5.34-1060$ & $y=8.8227 x+9.0811$ & 0.9999 \\
\hline
\end{tabular}

a) $y$ denotes the peak areas and $x$ denotes the concentrations

Table 9. Recovery Study for Compounds $\mathbf{1}-\mathbf{3}$

\begin{tabular}{|c|c|c|c|c|c|}
\hline Compound & Spiked (mg) & Level found (mg) $(\text { mean } \pm \text { S.D. })^{a}$ & Recovery (\%, mean \pm S.D. $)$ & RSD (\%) & Overall recovery $(\%$, mean \pm S.D. $)$ \\
\hline \multirow[t]{3}{*}{1} & 0.14 & $0.137 \pm 0.002$ & $98.0 \pm 1.81$ & 1.84 & $96.2 \pm 2.44$ \\
\hline & 0.28 & $0.262 \pm 0.006$ & $93.6 \pm 2.07$ & 2.21 & (RSD: $2.54 \%)$ \\
\hline & 0.56 & $0.543 \pm 0.002$ & $97.0 \pm 0.425$ & 0.60 & \\
\hline \multirow[t]{3}{*}{2} & 1.077 & $1.052 \pm 0.042$ & $97.7 \pm 3.95$ & 4.04 & $96.4 \pm 3.22$ \\
\hline & 2.154 & $2.103 \pm 0.064$ & $97.6 \pm 2.98$ & 3.05 & (RSD: $3.34 \%$ ) \\
\hline & 4.308 & $4.046 \pm 0.075$ & $93.9 \pm 1.73$ & 2.44 & \\
\hline \multirow[t]{3}{*}{3} & 0.084 & $0.087 \pm 0.002$ & $103.4 \pm 3.06$ & 2.96 & $100.5 \pm 3.17$ \\
\hline & 0.164 & $0.163 \pm 0.005$ & $99.6 \pm 2.99$ & 3.00 & (RSD: $3.16 \%)$ \\
\hline & 0.328 & $0.323 \pm 0.003$ & $98.4 \pm 1.01$ & 1.42 & \\
\hline
\end{tabular}

a) Standard deviation $(n=3)$

traction efficiency (Table 7). Based on these findings, the conditions of extracting with $80 \%$ methanol using sonication for twice was applied for subsequent extraction of compounds 1, 2 and $\mathbf{3}$ from the herbal materials. The extraction efficiency of compounds $\mathbf{1}-\mathbf{3}$ was found to be larger than $98 \%$ by optimizing the extraction solvents, time and methods.

Method Validation Linearity was examined with a series of standard solution in the concentration range of 5$1000 \mu \mathrm{g} / \mathrm{ml}$. Satisfactory linearity of each compound was obtained (Table 8).

Method precision was investigated by repeatedly analyzing the same set of standard solution, with the values of relative standard deviations (RSDs) for compounds 1, 2 and 3, reported as $0.72,0.72$ and $0.75 \%(n=6)$, respectively.

Method reproducibility was evaluated by six replicated analyses of herbal samples. The RSDs of the content of compounds $\mathbf{1}-\mathbf{3}$ in six replicated herbal samples was $3.26,1.78$ and $1.83 \%$, respectively.

Stability testing was performed on a standard stock solution over a period of fifteen days and sample solution after standing at time intervals of $0,2,4,6,8,12 \mathrm{~h}$ in a single day and further keeping for 1 and $2 \mathrm{~d}$. The results showed that the RSDs of compounds $\mathbf{2}-\mathbf{3}$ in the standard solution was 2.35 and $0.77 \%(n=6)$ in $15 \mathrm{~d}$, respectively. However, the RSD of compound 1 in the standard solution was $2.23 \%(n=3)$ in three consecutive days but $8.08 \%(n=6)$ in $15 \mathrm{~d}$. For the sta- bility of sample solution, the reported RSDs of the compounds $1-3$ were found to be $2.12,0.38$ and $0.50 \%(n=6)$ over the period of $12 \mathrm{~h}$ as well as $1.71,0.18$ and $0.25 \%$ $(n=3)$ over the period of three days, respectively. As a result, compounds $\mathbf{1}-\mathbf{3}$ were relatively stable in methanol and $80 \%$ methanol for at least $3 \mathrm{~d}$.

Recovery study was conducted on a sample spiked with different concentration levels, namely 50, 100 and $150 \%$ of known amounts of compounds $\mathbf{1}-\mathbf{3}$ in the samples. The results showed that all the average recovery fell in the range of 96.2-100.5\% with average RSDs 3.01\% $(n=9)$ (Table 9).

The limit of detection, evaluated by a signal-to-noise ratio of about $3: 1$ for the standard solution, was determined to be $0.528,0.268$ and $0.267 \mu \mathrm{g} / \mathrm{ml}$ for compounds $\mathbf{1}, \mathbf{2}$ and $\mathbf{3}$, respectively.

The above results were considered to be satisfactory for subsequent quantitative analysis of herbal samples.

Quantification of Herb Samples As discussed earlier, both substituted species of $O$. corymbosa and $O$. tenelliflora were also used under the name of Herba Oldenlandiae in the herbal market. Therefore, the contents of compounds $1-\mathbf{3}$ (Table 1) in 43 samples derived from three species of $O$. diffusa, $O$. corymbosa and $O$. tenelliflora which collected and purchased form different geographic areas were determined using the above analytical method. Compounds 1, 2 and 3 were well resolved in the HPLC chromatograms with elution time at 6.81, 19.5 and 36.4 min, respectively (Fig. 3). 


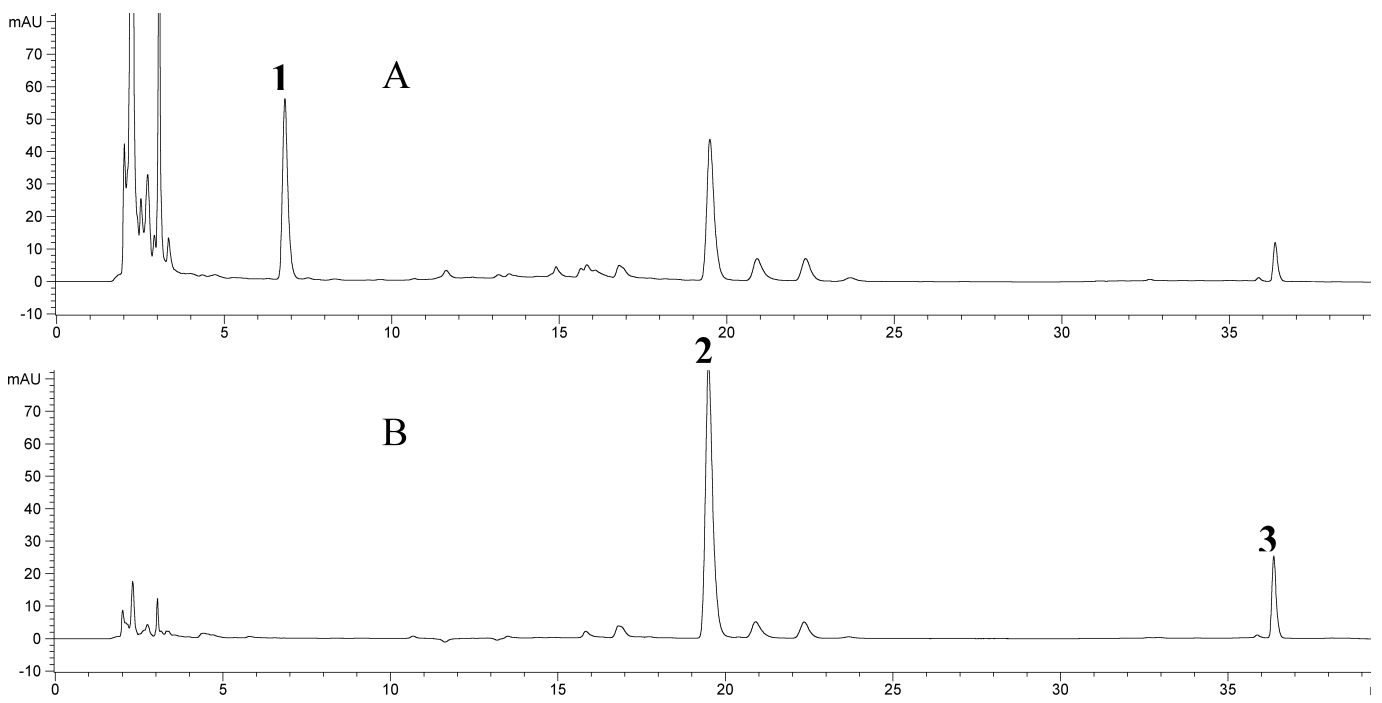

Fig. 3. Typical HPLC Chromatogram of Herba Oldenlandiae

(A) Detection wavelength $=238 \mathrm{~nm}$; (B) detection wavelength $=312 \mathrm{~nm}$.

In this study, the results showed that $O$. diffusa containing all the three compounds, compound 1 was often found in all three species. Both compounds $\mathbf{2}$ and $\mathbf{3}$ can only be found in a few samples of $O$. corymbosa, but totally absent in O. tenelliflora. From Table 1, the contents of compounds 1-3 of $O$. diffusa were $2.476 \pm 2.804,4.166 \pm 1.703$ and $0.791 \pm 0.509 \mathrm{mg} / \mathrm{g}(n=23)$, respectively, which were significantly higher than that in $O$. corymbosa (Table 2). Based on the existence of compounds $\mathbf{1}-\mathbf{3}$, it can be fairly straightforward to differentiate $O$. diffusa from the substitutes of $O$. corymbosa and $O$. tenelliflora. Moreover, those indicated that compounds $\mathbf{1}-\mathbf{3}$ were better and representative markers than ursolic acid and oleanolic acid for the identification and quality assessment of Herba Oldenlandiae.

On the other hand, the content of compounds $\mathbf{1}-\mathbf{3}$ from different sources was found vary greatly within the same species. It is well-known that Guangdong, Guangxi and Fujian Provinces in China are the major production areas of Herba Oldenlandiae. However, the contents of compounds $\mathbf{1}-\mathbf{3}$ in the samples of $O$. diffusa collected from the above areas were found to be markedly different. The content of compound $\mathbf{3}$ was found relatively lower in Fujian's samples. In contrast, most samples collected from Guangdong province contained higher contents of compound 3. However, the contents of compounds $\mathbf{1} \mathbf{- 2}$ in Guangdong's samples $(1.902 \pm 1.699 \mathrm{mg} / \mathrm{g}$ for compound 1 and $4.236 \pm 1.244 \mathrm{mg} / \mathrm{g}$ for compound 2, $n=10$ ) were lower than those in Fujian's samples $(4.158 \pm 4.877 \mathrm{mg} / \mathrm{g}$ for compound 1 and $6.267 \pm$ $0.827 \mathrm{mg} / \mathrm{g}$ for compound $\mathbf{2}, n=3$ ). Besides, the content of compound $\mathbf{2}$ is always the highest among other iridoid contents in $O$. diffusa.

Eight batches of Herba Oldenlandiae and five batches of O. corymbosa were respectively collected in the same herbal market of Guangdong and Hong Kong in different periods. By using the developed analytical method and rationale developed, four batches of collected samples from Guangdong were authenticated to be $O$. corymbosa. It is worthnoting that the contents of compounds $\mathbf{1}-\mathbf{3}$ were found varied greatly in these samples collected from the same market. Therefore, more attention should be paid in the proper use of Herba
Oldenlandiae.

\section{Conclusion}

A convenient method has been developed for the quantitative analysis of the contents of three iridoid glucosides in Herba Oldenlandiae. The validation data indicated that this method is reliable. Satisfactory chromatographic separation of compounds $\mathbf{1}-\mathbf{3}$ with their adjacent peaks could be obtained by using two UV absorption wavelengths at 238 and $312 \mathrm{~nm}$. The extraction efficiency of compounds $\mathbf{1}-\mathbf{3}$ were optimized by selecting appropriate extraction solvents, time and methods.

Conventionally, ursolic acid and oleanolic acid were commonly used as marker compounds for the quality assessment of Herba Oldenlandiae. In this paper, three iridoid glucosides have been used as chemical markers for the first time to evaluate the quality of Herba Oldenlandiae. The analytical results here showed that compound 1 was the first time found in $O$. tenelliflora whilst compounds $\mathbf{2}$ and $\mathbf{3}$ were reported in $O$. corymbosa and $O$. tenelliflora. Moreover, the contents of compounds $\mathbf{1}-\mathbf{3}$ were significantly varied among samples whilst the levels of compounds $\mathbf{2}$ and $\mathbf{3}$ were found to be in lower contents in the two substitutes than those of $O$. diffusa. Therefore, it is crucial to use the three iridoid glucosides as chemical markers for the differentiation from substitutes and evaluation on the quality of Herba Oldenlandiae.

Acknowledgments The project was supported by the Faculty Research Grant of Hong Kong Baptist University (FRG/04-05/II-55).

\section{References}

1) Xu G. J., Xu L. S., Wang Z. T., "Species Systematization and Quality Evaluation of Commonly Used Chinese Traditional Drugs (SouthChina Edition)," Vol. IV, Fujian Science and Technology Press, Fuzhou, 1997, p. 658

2) "The State Pharmacopoeia Committee of China, The Appendix of Chinese Pharmacopoeia," Vol. I, Chemical Industry Publishing House, Beijing, 2005, p. 22.

3) Zhao Z. Z., Li T. Y. S., "Authentication of Easily Confused Chinese Materia Medica in Hong Kong," Published by the Chinese Medicine Merchants Association Ltd., Hong Kong, 2004, p. 144.

4) Nishihama Y., Masuda K., Yamaki M., Takagi S., Sakina K., Planta Med., 43, 28-33 (1981). 
5) Wu H., Tao X., Chen Q., Lao X., J. Nat. Prod., 54, 254-256 (1991).

6) Lu H. C., He J., Natural Product Research and Development, 8, 34 37 (1996).

7) Kim Y., Park E. J., Kim J., Kim Y., Kim S. R., Kim Y. Y., J. Nat. Prod., 64, 75-78 (2001).

8) Lu C. M., Yang J. J., Wang P. Y., Lin C. C., Planta Med., 66, 374-377 (2000).

9) Ren F. Z., Liu G. S., Zhang L., Niu G. Y., Chin. Pharm. J., 40, 502504 (2005).

10) Wu K. S., Zhang K., Tan G. S., Zeng G. Y., Zhou Y. J., Chin. Pharm. $J ., 40,817-819$ (2005).

11) Kupeli E., Harput U. S., Varel M., Yesilada E., Saracoglu I., J. Ethnopharmacol., 102, 170-171 (2005).

12) Park K. S., Chang I. M., Planta Med., 70, 778-779 (2004).

13) Suksamrarn A., Kumpun S., Kirtikara K., Yingyongnarongkul B., Suksamrarn S., Planta Med., 68, 72-73 (2002).

14) Su B. N., Pawlus A. D., Jung H. A., Keller W. J., McLaughlin J. L., Kinghorn A. D., J. Nat. Prod., 68, 592-595 (2005).

15) Koo H. J., Lee S., Shin K. H., Kim B. C., Lim C. J., Park E. H., Planta
Med., 70, 467-469 (2004).

16) Pungitore C. R., Ayub M. J., Garcia M., Borkowski E. J., Sosa M. E., Ciuffo G., Giordano O. S., Tonn C. E., J. Nat. Prod., 67, 357-361 (2004).

17) Li Y., Ishibashi M., Satake M., Oshima Y., Ohizumi Y., Chem. Pharm. Bull., 51, 1103-1105 (2003).

18) Misra A. P., Mathad V. T., Raj K., Bhaduri A. P., Tiwari R., Srivastava A., Mehrotra P. K., Bioorg. Med. Chem., 9, 2763-2772 (2001).

19) Zhou C., Wang L., Feng X. Y., Chinese Medicinal Materials, 25, $313-314$ (2002).

20) Luo X. G., Jiang L. L., Zhao S. J., China Pharmacy, 15, 175-176 (2004).

21) Zhang C. H., Guo X. J., Li F., Xie X. F., Li F. M., J. Shenyang Pharmaceutical University, 21, 358-360 (2004).

22) Ou M. R., Xu X. P., Deng Y. J., Xu C. J., Strait Pharmaceutical J., 16, $43-45$ (2004).

23) Zhang C. H., Guo X. J., Xue X. F., Yang L., Ran G. M., Li F. M., Chin. Pharm. J., 39, 854-855 (2004). 